\title{
POSITION OF SINGULARITIES AND FUNDAMENTAL GROUP OF THE COMPLEMENT OF A UNION OF LINES
}

\author{
KWAI-MAN FAN
}

(Communicated by Peter Li)

\begin{abstract}
In this paper we give two examples of complex line arrangements in $C P^{2}$ with 7 lines, that both have 3 triple points and 12 double points, and their complements have nonisomorphic global fundamental groups. These two line arrangements are, in some sense, a much simpler example of a pair of plane algebraic curves that have the same local topology but have complements with different global topology - compare with the example given by Zariski, or the recent example given by Artal-Bartolo.
\end{abstract}

\section{INTRODUCTION}

We are interested in the question of the dependence between the topology of the complement of a complex plane curve and the position of the singularities. In [7], Zariski showed that the fundamental group of the complement of an irreducible sextic curve in the projective plane with six cusps lying on a conic is isomorphic to the free product of $Z_{2}$ and $Z_{3}$ (see also [4]). In [8], he then showed that the complement of an irreducible sextic curve with six cusps that do not lie on a conic has abelian fundamental group. This is the first pair of curves known to have identical local topology but to have complements with different global topology. Recently, in 1990, Artal-Bartolo constructed a pair of complex line arrangements in the projective plane that have the same local topology, both have 9 lines, 9 triple points, and their complements have different Alexander polynomials [1] (see also [2], 212-213).

The two examples of line arrangements given by Artal-Bartolo are as follow: the first arrangement $V_{1}$ is given by

$$
\begin{gathered}
x y z(x-y)(y+b z)(x-y-z)(a x+y+z)(a x+y+b z) \\
\times(a b x+(a-a b+1) y+b z)=0,
\end{gathered}
$$

where $a b(a+1)(b-1)(a+b)(a-a b+1)(a b+b-1) \neq 0$.

The second line arrangement $V_{2}$ is given by

$$
\begin{gathered}
x y z(x+y)(x+a z)(y+b z)(c x+(c+1) y+b z) \\
\times(c(a+b) x+a(c+1) y+a b(c+1) z)(c x+(c+1) y+a c z)=0,
\end{gathered}
$$

Received by the editors May 1, 1995.

1991 Mathematics Subject Classification. Primary 14H30; Secondary 14H20.

(C)1996 American Mathematical Society 
where $a b c(c+1)(a c-b)(a+b) \neq 0$ and $c(a-b c)-b(c+1)=0$. It was shown in [1] (see also [2]) that the Alexander polynomials $\Delta_{V_{i}}(t)$ of the curves $V_{i}, i=1,2$, are:

$$
\begin{gathered}
\Delta_{V_{1}}(t)=(t-1)^{8}\left(t^{2}+t+1\right), \\
\Delta_{V_{2}}(t)=(t-1)^{8} .
\end{gathered}
$$

These two examples are used to exhibit a change in the first Betti number of the corresponding Milnor fiber. It is believed that the example of Artal-Bartolo is among the simplest examples showing the dependence of the topology of the complement of a curve on the position of the singularities.

By studying the fundamental groups of some line arrangements that have less than 8 lines, the author found a pair of line arrangements with 7 lines, that both have 3 triple points and 12 double points and their complements have nonisomorphic global fundamental groups. This provides a simpler pair of line arrangements that have the same local topology but have complements with different global topology.

In section 2, we shall describe a product theorem of a reducible curve of M. Oka and Sakamoto [5] and use it to study the fundamental groups of complements of some line arrangements. In section 3 , we shall give two line arrangements that both have 7 lines, 3 triple points and 12 double points, and then study the fundamental groups of their complements in $C P^{2}$. We shall show that the fundamental group of the complement of the first example of line arrangement is isomorphic to the direct product of 3 copies of free groups on two generators, hence has a trivial center. On the other hand, the fundamental group of the complement of the second example of line arrangement has a nontrivial center. Hence these two groups cannot be isomorphic.

\section{2. $\pi_{1}$ OF COMPLEMENTS OF SOME LINE ARRANGEMENTS}

We are going to use the following result of Oka and Sakamoto [5]:

Theorem 2.1. Let $C_{1}$ and $C_{2}$ be two distinct algebraic curves in the complex affine plane $C^{2}$ of degree $n_{1}$ and $n_{2}$ respectively. Suppose that $C_{1}$ and $C_{2}$ intersect at $n_{1} n_{2}$ distinct points. Then $\pi_{1}\left(C^{2} \backslash\left(C_{1} \cup C_{2}\right)\right) \cong \pi_{1}\left(C^{2} \backslash C_{1}\right) \oplus \pi_{1}\left(C^{2} \backslash C_{2}\right)$.

Denote a free group on $t$ generators by $F_{t}$. Let $\Sigma_{0}=\bigcup_{i=1}^{n} L_{i}$ be a union of $n$ distinct projective lines in the projective plane $C P^{2}$ such that all $L_{i}$ pass through a point $O$, and $C^{2}=C P^{2} \backslash L_{n}$. If we transform the line $L_{n}$ to become the line at infinity to $C^{2}$, then $C^{2} \cap\left(\bigcup_{i=1}^{n-1} L_{i}\right)$ is a set of $n-1$ parallel lines, and it is easy to see that $\pi_{1}\left(C P^{2} \backslash \Sigma_{0}\right) \cong \pi_{1}\left(C^{2} \backslash\left(C^{2} \cap\left(\bigcup_{i=1}^{n-1} L_{i}\right)\right)\right) \cong F_{n-1}$. Denote the multiplicity of a point $P$ of a curve by $m(P)$. By using Theorem 2.1, we have:

Theorem 2.2. Let $\Sigma=\bigcup_{i=1}^{n} L_{i}$ be a line arrangement in $C P^{2}$ and assume that there is a line $L$ of $\Sigma$ such that for any singular point $S$ of $\Sigma$ with $m(S) \geq 3$, we have $S \in L$. Then $\pi_{1}\left(C P^{2} \backslash \Sigma\right)$ is isomorphic to a direct product of free groups.

Proof. Let $\left\{S_{1}, S_{2}, \cdots, S_{t}\right\}$ be the set of all singular points of $\Sigma$ with $m\left(S_{i}\right) \geq 3$; then, by our assumption, $\left\{S_{1}, \cdots, S_{t}\right\} \subset L$. Let the number of nodes of $\Sigma$ that lie on $L$ be $r$, and note that $n=r+1-t+\sum_{i=1}^{t} m\left(S_{i}\right)$.

Suppose $t=0$; then $\Sigma$ is $r+1$ lines in general position and $\pi_{1}\left(C P^{2} \backslash \Sigma\right) \cong A_{r}$, where $A_{r}$ is a free abelian group on $r$ generators [7].

Suppose that $t>0$. Let $C^{2}=C P^{2} \backslash L$; then $C P^{2} \backslash \Sigma=C^{2} \backslash(\Sigma \backslash L)$. Note that $(\Sigma \backslash L) \cap C^{2}=\sigma_{0} \cup \sigma_{1} \cup \cdots \cup \sigma_{t}$, where 
a) $\sigma_{0}$ is $r$ lines in general position,

b) $\sigma_{i}, i>0$, is $m\left(S_{i}\right)-1$ distinct parallel lines in $C^{2}$.

Lines in $\sigma_{i}$ and $\sigma_{j}$ intersect if $i \neq j, 0 \leq i, j \leq t$, and $(\Sigma \backslash L) \cap C^{2}$ has only nodes. Note that the fundamental group of the complement of $k$ parallel lines in $C^{2}$ is isomorphic to a free group on $k$ generators. By Theorem 2.1, we have:

$$
\begin{gathered}
\pi_{1}\left(C^{2} \backslash\left(\sigma_{0} \cup \sigma_{1} \cup \cdots \cup \sigma_{t}\right)\right. \\
\cong \pi_{1}\left(C^{2} \backslash \sigma_{0}\right) \oplus \pi_{1}\left(C^{2} \backslash \sigma_{1}\right) \oplus \cdots \oplus \pi_{1}\left(C^{2} \backslash \sigma_{t}\right) \\
\cong A_{r} \bigoplus_{i=1}^{t} F_{m\left(S_{i}\right)-1}
\end{gathered}
$$

Note that in the above proof, we actually showed that:

Corollary 2.1. Let $E$ be the set $\left\{S_{1}, S_{2}, \cdots, S_{t}\right\}$ of all singular points of $\Sigma$ with multiplicity $\geq 3$, and the number of nodes of $\Sigma$ on $L$ be $r$. Suppose that the line arrangement $\Sigma$ has a component $L$ such that $E \subset L$. Then

$$
\pi_{1}\left(C P^{2} \backslash \Sigma\right) \cong A_{r} \bigoplus_{i=1}^{t} F_{m\left(S_{i}\right)-1},
$$

where $A_{r}$ is a free abelian group on $r$ generators and $m\left(S_{i}\right)$ is the multiplicity of $S_{i}$.

A line arrangement that satisfies the conditions of Corollary 2.1 can be constructed easily. For example, take $L$ to be the $X$-axis, and choose 3 points $S_{1}, S_{2}, S_{3}$ on $L$, and at each $S_{i}$, choose two distinct lines $L_{1}^{i}, L_{2}^{i} \neq L$ that go through $S_{i}$, and require that these lines intersect in nodes at points that do not lie on $L$. The union of these seven lines gives a line arrangement in $C P^{2}$, and we call it $\Sigma^{\prime}$. By Corollary 4.1 , we have $\pi_{1}\left(C P^{2} \backslash \Sigma^{\prime}\right) \cong F_{2} \oplus F_{2} \oplus F_{2}$.

We are going to use the following lemma (cf. [9]) in the next section:

Lemma 2.1. Let $\Sigma=L_{1} \cup L_{2} \cup \cdots \cup L_{n}, n>1$, be a line arrangement in $C P^{2}$ with $n$ components. Suppose that $L_{1}$ intersects $L_{2} \cup \cdots \cup L_{n}$ at $n-1$ distinct points. Then $\pi_{1}\left(C P^{2} \backslash \Sigma\right)$ splits as a direct product of two groups $Z$ and $H$, where $Z$ is an infinite cyclic group on one generator.

Proof. Let $C^{2}=C P^{2} \backslash L_{n}$; then $L_{1} \cap C^{2}$ intersects $\left(L_{2} \cup \cdots \cup L_{n-1}\right) \cap C^{2}$ at $n-2$ distinct points. By Theorem 2.1, we have

$$
\begin{gathered}
\pi_{1}\left(C P^{2} \backslash \Sigma\right) \cong \pi_{1}\left(C^{2} \backslash\left(L_{1} \cup L_{2} \cup \cdots \cup L_{n-1}\right) \cap C^{2}\right) \\
\cong \pi_{1}\left(C^{2} \backslash L_{1} \cap C^{2}\right) \oplus \pi_{1}\left(C^{2} \backslash\left(L_{2} \cup \cdots \cup L_{n-1}\right) \cap C^{2}\right) \cong Z \oplus H,
\end{gathered}
$$

where $Z=\pi_{1}\left(C^{2} \backslash L_{1} \cap C^{2}\right)$ is an infinite cyclic group on one generator, and $H=\pi_{1}\left(C^{2} \backslash\left(L_{2} \cup \cdots \cup L_{n-1}\right) \cap C^{2}\right)$. 


\section{Two Line ARRANGEMENTS With 7 LINES}

Let $\Sigma=\bigcup_{i=1}^{n} L_{i}$ be a line arrangement in $C P^{2}$, and $\left\{A_{1}, A_{2}, \cdots, A_{b}\right\}$ be the set of all singular points of $\Sigma$. Then by counting intersection numbers, we have:

$$
\frac{n(n-1)}{2}=\sum_{i=1}^{b} \frac{m\left(A_{i}\right)\left(m\left(A_{i}\right)-1\right)}{2} .
$$

Below we are going to give two line arrangements with 7 lines that have the same local topology but have complements with different global topology.

$I$. Let $W_{1}$ be a line arrangement in $C P^{2}$ given by the following homogeneous equation:

$$
y(x-y)(x+y)(2 x-y-2 z)(2 x+y-2 z)(3 x-y-6 z)(3 x+y-6 z)=0 .
$$

Put $z=1$; we see that $W_{1}$ is given by the $X$-axis, together with 3 pairs of lines such that each pair intersects the $X$-axis at a triple point, and $W_{1}$ has 3 triple points and 12 double points. Coordinates of triple points are: $[x, y, z]=$ $[0,0,1],[1,0,1],[2,0,1]$. Coordinates of double points are: $[2,2,1],\left[\frac{2}{3}, \frac{2}{3}, 1\right],[3,3,1]$, $\left[\frac{3}{2}, \frac{3}{2}, 1\right],[4,6,1],\left[\frac{8}{5}, \frac{6}{5}, 1\right],[2,-2,1],\left[\frac{2}{3}, \frac{-2}{3}, 1\right],[3,-3,1],\left[\frac{3}{2}, \frac{-3}{2}, 1\right],[4,-6,1],\left[\frac{8}{5}, \frac{-6}{5}, 1\right]$. By Corollary 2.1, we have

$$
\pi_{1}\left(C P^{2} \backslash W_{1}\right) \cong F_{2} \oplus F_{2} \oplus F_{2} .
$$

$I I$. Let $W_{2}$ be a line arrangement in $C P^{2}$ given by the following homogeneous equation:

$$
x y(x-y)(x+y-2 z)(x-2 y-2 z)(2 x+y-2 z)(3 x-y-9 z)=0 .
$$

Put $z=1$. We find that coordinates of triple points are: $[x, y, z]=[0,0,1],[2,0,1]$, $[0,2,1]$. Coordinates of double points are: $[1,0,1],[0,-1,1],[1,1,1],[-2,-2,1]$, $\left[\frac{2}{3}, \frac{2}{3}, 1\right],\left[\frac{6}{5}, \frac{-2}{5}, 1\right],[3,0,1],[0,-9,1],\left[\frac{9}{2}, \frac{9}{2}, 1\right],\left[\frac{11}{4}, \frac{-3}{4}, 1\right],\left[\frac{11}{5}, \frac{-12}{5}, 1\right],\left[\frac{16}{5}, \frac{3}{5}, 1\right]$. Note that the component of $W_{2}$ given by $3 x-y-9 z=0$ intersects all other lines at normal crossings, hence by Lemma 2.1 we have

$$
\pi_{1}\left(C P^{2} \backslash W_{2}\right) \cong Z \oplus N
$$

where $Z$ is an infinite cyclic group on one generator. In particular, this shows that $\pi_{1}\left(C P^{2} \backslash W_{2}\right)$ has a nontrivial center.

Theorem 3.1. There exist two line arrangements with 7 lines in $C P^{2}$ that both have 3 triple points and 12 double points, yet the fundamental groups of their complements are not isomorphic.

Proof. By (3.1), we have $G_{1}=\pi_{1}\left(C P^{2} \backslash W_{1}\right) \cong F_{2} \oplus F_{2} \oplus F_{2}$. We see that the center of $G_{1}$ is trivial since the free group $F_{2}$ has a trivial center. On the other hand, (3.2) implies that $G_{2}=\pi_{1}\left(C P^{2} \backslash W_{2}\right)$ has a nontrivial center, hence $G_{1}$ and

$G_{2}$ cannot be isomorphic.

\section{REFERENCES}

1. E. Artal-Bartolo, Sur le premier nombre de Betti de la fibre de Milnor du cone sur une courne projective plane et son rapport avec la position des points singuliers, University of Wisconsin, Madison (preprint 1990).

2. A. Dimca, Singularities and Topology of Hypersurfaces, Springer-Verlag, New York (1992). MR 94b:32058 
3. E. R. van Kampen, On the fundamental group of an algebraic curve, Amer. J. Math. 55 (1933), 255-260.

4. M. Oka, Some plane curves whose complements have non-abelian fundamental groups, Math. Ann. 218 (1975), 55-65. MR 53:419

5. M. Oka and K. Sakamoto, Product theorem of the fundamental group of a reducible curve, J. Math. Soc. Japan 30 (1978), 599-602. MR 81h:14019

6. R. Randell, The fundamental group of the complement of a union of a complex hyperplanes, Invent. Math. 80 (1985), 467-468, Correction: Invent. Math. 80 (1985), 467-468. MR 87e:32010

7. O. Zariski, On the problem of existence of algebraic functions of two variables possessing a given branch curves, Amer. J. Math. 51 (1929), 305-328.

8. O. Zariski, The topological discriminant group of a Riemann surface of genus p, Amer. J. Math. 59 (1937), 335-358.

9. O. Zariski, On the irregularity of cyclic multiple plane, Ann. of Math. 32 (1931), 485-511.

Department of Mathematics, National Chung Cheng University, Minghsiung, Chiayi 621, TAIWAN

E-mail address: kmfan@math.ccu.edu.tw 